\title{
The Neuroprotective Effect and Probable Mechanism of DL-3-n-Butylphthalide in Brain Diseases
}

\author{
Yinyi Sun ${ }^{a} \quad$ Kangyong Liu ${ }^{a}$ b $\quad$ Ting Zhang $^{a} \quad$ Yanmeng Sun $^{a}$ Wenqi Chen $^{a}$ \\ Qian $\mathrm{Li}^{\mathrm{a}}$ Rongfu Chen ${ }^{\mathrm{a}}$ Xiaojiang Sun ${ }^{\mathrm{a}}$ \\ a Department of Neurology, Shanghai Jiaotong University Affiliated Sixth People's Hospital, and \\ ${ }^{b}$ Department of Neurology, Shanghai Pudong New Area Zhoupu Hospital, Shanghai, China
}

\section{Key Words}

3-n-Butylphthalide · Neuroprotective effect · Parkinson's disease · Alzheimer's disease ·

Cerebral ischemia

\section{Abstract}

3-n-Butylphthalide (NBP), one of the chemical constituents of celery oil, is used for the treatment of many central nervous system diseases, including Parkinson's disease, Alzheimer's disease and cerebral ischemia. NBP showed potent neuroprotective effects by decreasing oxidative damage, inhibiting inflammatory responses, improving mitochondrial function, and reducing neuronal apoptosis.

(C) 2014 S. Karger AG, Basel

\section{Introduction of 3-n-Butylphthalide}

3-n-Butylphthalide (NBP), an extract from the seeds of Apium graveolens Linn (Chinese celery), was synthesized and received approval by the State Food and Drug Administration of China for clinical use in stroke patients in 2002 [1]. NBP is a chiral compound, which contains both L- and D-isomers. Peng et al. [2] found that L-3-n-butylphthalide (L-NBP) attenuated learning and memory deficits induced by chronic cerebral hypoperfusion in rats.

NBP is a new drug which could increase the blood flow of the ischemic area to improve energy metabolism [3], promote microvascular regeneration to reconstruct ischemic microcirculation [4, 5], and protect the mitochondria to rescue ischemic penumbra cells. In addi- 
tion, NBP could reduce the infarct volume, local cerebral ischemia, brain edema and inflammation in animal models [6]. It also has antiplatelet aggregation and antithrombotic effects [7]. Here we provide a brief review of the roles of NBP in Parkinson's disease (PD), Alzheimer's disease (AD) and cerebral ischemia. We also discuss the controversies and challenges in exploiting NBP as a therapeutic strategy.

\section{The Mechanisms of the Neuroprotective Effect of NBP}

The probable mechanisms of NBP may involve: (1) anti-apoptotic effects - NBP plays the role of anti-apoptosis through a mitochondrion-related caspase-dependent or nondependent apoptotic pathway [8-11]; (2) anti-oxidative stress - NBP could inhibit mitochondrial permeability, increase the cellular GSH content, and inhibit the overproduction of nitric oxide to play the role of anti-oxidative stress [1], and (3) improve learning and memory deficits - NBP can reduce abnormal phosphorylation of tau protein by downregulating GSK-3 $\beta$ activity [12] or increase alpha-amyloid precursor protein ( $\alpha \mathrm{APP}$ ) release by increasing $\mathrm{PKC} \alpha, \mathrm{PKC} \varepsilon$ activity and MAPK phosphorylation levels to reduce $\beta$-amyloid protein $(A \beta)$ generation [13]. Pretreatment with L-NBP could significantly increase cell viability of $\mathrm{H}_{2} \mathrm{O}_{2}$-damaged cells and reduce $\mathrm{H}_{2} \mathrm{O}_{2}$-induced neuronal apoptosis. NBP treatment at a dose of $10 \mu \mathrm{M}$ inhibited $\mathrm{H}_{2} \mathrm{O}_{2}$ induced downregulation of Bcl-2, Bcl-w, and PKC $\alpha$ but also attenuated the overexpression of Bax. The PKC inhibitor calphostin C significantly attenuated the protective effects of NBP. This suggested that NBP might protect neurons against $\mathrm{H}_{2} \mathrm{O}_{2}$-induced apoptosis by modulating apoptosis-related genes and activating the PKC $\alpha$ pathway [9]. Another study reported that vascular endothelial growth factor expression was upregulated, while the caspase- 3 expression was reduced in the diabetes mellitus-NBP rats. It showed that NBP may have a protective effect on diabetic brain damage through enhancing vascular endothelial growth factor expression to inhibit caspase-3-mediated apoptosis [10]. In conclusion, NBP is a multitarget clinical drug, suggesting that it may have therapeutic potential for central nervous system diseases.

\section{The Effect of NBP in PD}

The clinical features of PD are resting tremor, muscle rigidity, bradykinesia and postural reflex disorder. Motor symptoms of PD are mainly due to the selective degeneration of substantia nigra dopaminergic neurons in the ventral midbrain, which results in a decrease in dopamine levels in the striatum [14]. $\alpha$-Synuclein oligomers and aggregates play an important role in the pathogenesis and development process of PD $[1,15]$. Protein accumulations in cytoplasmic inclusions are called Lewy bodies. Our previous studies have shown that MPTP-induced oxidative stress promoted the oligomerization of $\alpha$-synuclein that leads to the pathological aggregation of $\alpha$-synuclein [15].

The present study demonstrated that NBP might be promising for the clinical treatment of patients with PD. NBP can protect against $\mathrm{MPP}^{+}$-induced neurotoxicity via activation of an autophagic process $[1,15]$. This report strongly suggests that NBP promotes intracellular autophagic activity and subsequently enhances the degradation of $\alpha$-synuclein. Thus, it can be concluded that autophagy protects against PD through self-digestion of mis-aggregated proteins, such as $\alpha$-synuclein [16]. It has been found that NBP has the ability to suppress the release of cytochrome $\mathrm{C}$, to stimulate the upregulation of vascular endothelial growth factor, and subsequently to protect against oxidative stress in diabetic rats $[10,16]$. 
Similarly, NBP administration reduces oxidative stress in PD models. It does this by accentuating the expression of the vesicular monoamine transporter 2 genes and protecting dopaminergic neuronal tissue by inhibiting oxidative stress $[17,18]$. It also ameliorates mitochondrial dysfunction seen in PD [1].

\section{The Effect of NBP in AD}

$\mathrm{AD}$ is the most common form of dementia in the elderly and has a variety of clinical symptoms. Its main pathological feature appears to be $A \beta$ deposits in the brain, including senile plaques and neurofibrillary tangles [19]. $A \beta$ is thought to be a key player in neuronal damage and dementia in AD patients. $A \beta$ is a fragment from a larger protein called APP, a transmembrane protein that penetrates through the neuron's membrane. APP is critical to neuron growth, survival and postinjury repair [20].

The mechanism of NBP improving cognitive impairment remains unclear. In a recent study, it was found that NBP ameliorated the impairment of spatial learning and working memory in an $A \beta$-infused model [12]. The effect may be related to enhanced $A \beta$ clearance and degradation. Moreover, it was reported that NBP increased insulin-degrading enzyme activity in a triple transgenic mouse model carrying human mutant APP, presenilin 1 , and tau transgenes [12]. Another report showed that the effect by NBP of lowering cerebral A $\beta$ accumulation may be attributable to directing APP processing toward a nonamyloidogenic pathway [21].

Oxidative damage plays an important role in the development and progression of AD. A $\beta$ directly interacts with mitochondria, and then induces the generation of free radicals, mitochondrial dysfunction and cell death [22]. It was shown that NBP reversed A $\beta$-induced oxidative injuries. NBP appears to be an effective anti-oxidant. Glutathione peroxidase is one of the main enzymes involved in the cellular protection against damage from oxygen-derived free radicals $[12,23]$. Glutathione peroxidase may be an important factor in $A \beta$-induced oxidative stress. In addition, it may be a target of NBP for preventing $A \beta$-induced neuronal damage from superoxidation [12].

\section{The Effect of NBP in Cerebral Ischemia}

Cerebral ischemia is induced when there is not enough blood flowing to the brain; in this case, the blood cannot meet the metabolic needs. When the brain is hypoxic, we found that the cerebral metabolism changes and the metabolic rate decreases. This results in the death of brain tissue or ischemic stroke [24].

NBP has the ability to decrease the area of cerebral infarct in focal cerebral ischemic rats [25]. It can also improve energy metabolism in mice with complete brain ischemia [26]. The positive effects of NBP and L-NBP on cerebral ischemia and cerebral infarct have been verified in ischemic patients and animal models; however, little is known about the neuropotective machinery of NBP.

Mitochondria play a crucial role in apoptosis. NBP also increases the ATP level in rats with cerebral ischemia, and therefore prevents mitochondrial dysfunction resulting from ATP depletion [27]. Xiong and Feng [28] found that NBP improved mitochondrial dysfunction during cerebral ischemia. 
Sun et al.: The Neuroprotective Effect and Probable Mechanism of

DL-3-n-Butylphthalide in Brain Diseases

\section{Conclusion}

In summary, NBP showed neuroprotective effects by decreasing oxidative damage [29], inhibiting inflammatory responses [6], improving mitochondrial function, and reducing neuronal apoptosis [30]. The neuroprotective effects of NBP have potential therapeutic effects on the treatment of PD, AD and cerebral ischemia patients.

\section{Acknowledgements}

This work was supported by grants from the project of National Natural Science Foundation of China (No. 31171014 and No. 30970869), the project of Science and Technology Commission of Shanghai Municipality (No. 09DZ1950400) and the Board of Health of Shanghai, China (No. 2008086), the youth projects of the National Natural Science Foundation of China (No. 31100783) and the Youth Key Project in the Shanghai College of Medicine of Fudan University (No. 09-L37).

\section{References}

1 Huang JZ, Chen YZ, Su M, et al: dl-3-n-Butylphthalide prevents oxidative damage and reduces mitochondrial dysfunction in an MPP(+)-induced cellular model of Parkinson's disease. Neurosci Lett 2010;475:89-94.

-2 Peng Y, Xu S, Chen G, et al: 1-3-n-Butylphthalide improves cognitive impairment induced by chronic cerebral hypoperfusion in rats. J Pharmacol Exp Ther 2007;321:902-910.

-3 Chong Z, Feng Y: Protective effects of dl-3-n-butylphthalide on changes of regional cerebral blood flow and blood-brain barrier damage following experimental subarachnoid hemorrhage. Chin Med J (Engl) 1998;111: 858-860.

4 Liu CL, Liao SJ, Zeng JS, et al: dl-3n-butylphthalide prevents stroke via improvement of cerebral microvessels in RHRSP. J Neurol Sci 2007;260:106-113.

5 Liao SJ, Lin JW, Pei Z, et al: Enhanced angiogenesis with dl-3n-butylphthalide treatment after focal cerebral ischemia in RHRSP. Brain Res 2009;1289:69-78.

6 Xu HL, Feng YP: Inhibitory effects of chiral 3-n-butylphthalide on inflammation following focal ischemic brain injury in rats. Acta Pharmacol Sin 2000;21:433-438.

7 Wei W, Zhang W, Huang Y, et al: The therapeutic effect of (DL)-3-n-butylphthalide in rats with chronic cerebral hypoperfusion through downregulation of amyloid precursor protein and matrix metalloproteinase-2. J Int Med Res 2012;40:967-975.

-8 Cui YH, Zhang CD, Guo W, Wei YL: Effects of butylphthalide on the apoptosis of PC-12 cells under the induction of beta-amyloid peptide (in Chinese). Zhonghua Yi Xue Za Zhi 2010;90:3235-3237.

-9 Peng Y, Hu Y, Feng N, et al: L-3-n-butyl-phthalide alleviates hydrogen peroxide-induced apoptosis by PKC pathway in human neuroblastoma SK-N-SH cells. Naunyn Schmiedebergs Arch Pharmacol 2011;383:91-99.

-10 Zhang T, Jia W, Sun X: 3-n-Butylphthalide (NBP) reduces apoptosis and enhances vascular endothelial growth factor (VEGF) up-regulation in diabetic rats. Neurol Res 2010;32:390-396.

11 Li J, Li Y, Ogle M, et al: DL-3-n-butylphthalide prevents neuronal cell death after focal cerebral ischemia in mice via the JNK pathway. Brain Res 2010;1359:216-226.

12 Peng Y, Xing C, Xu S, et al: L-3-n-butylphthalide improves cognitive impairment induced by intracerebroventricular infusion of amyloid-beta peptide in rats. Eur J Pharmacol 2009;621:38-45.

-13 Peng Y, Hu Y, Xu S, et al: L-3-n-butylphthalide regulates amyloid precursor protein processing by PKC and MAPK pathways in SK-N-SH cells over-expressing wild type human APP695. Neurosci Lett 2011;487:211216.

14 Mythri RB, Bharath MM: Curcumin: a potential neuroprotective agent in Parkinson's disease. Curr Pharm Des 2012;18:91-99.

15 Liu K, Shi N, Sun Y, et al: Therapeutic effects of rapamycin on MPTP-induced Parkinsonism in mice. Neurochem Res 2013;38:201-207.

16 Liu K, Huang J, Chen R, et al: Protection against neurotoxicity by an autophagic mechanism. Braz J Med Biol Res 2012;45:401-407.

17 Kapoor S: Dl-3-n-butylphthalide and its emerging beneficial effects in neurology. Chin Med J (Engl) 2012;125: 3360.

18 Xiong N, Huang J, Chen C, et al: Dl-3-n-butylphthalide, a natural antioxidant, protects dopamine neurons in rotenone models for Parkinson's disease. Neurobiol Aging 2012;33:1777-1791.

19 Wang Y, Yin H, Wang L, et al: Curcumin as a potential treatment for Alzheimer's disease: a study of the effects of curcumin on hippocampal expression of glial fibrillary acidic protein. Am J Chin Med 2013;41:59-70. 
Sun et al.: The Neuroprotective Effect and Probable Mechanism of DL-3-n-Butylphthalide in Brain Diseases

20 Priller C, Bauer T, Mitteregger G, et al: Synapse formation and function is modulated by the amyloid precursor protein. J Neurosci 2006;26:7212-7221.

21 Peng Y, Sun J, Hon S, et al: L-3-n-butylphthalide improves cognitive impairment and reduces amyloid-beta in a transgenic model of Alzheimer's disease. J Neurosci 2010;30:8180-8189.

$\$ 22$ Reddy PH: Amyloid precursor protein-mediated free radicals and oxidative damage: implications for the development and progression of Alzheimer's disease. J Neurochem 2006;96:1-13.

23 Crack PJ, Cimdins K, Ali U, et al: Lack of glutathione peroxidase-1 exacerbates Abeta-mediated neurotoxicity in cortical neurons. J Neural Transm 2006;113:645-657.

24 Vespa P, Bergsneider M, Hattori N, et al: Metabolic crisis without brain ischemia is common after traumatic brain injury: a combined microdialysis and positron emission tomography study. J Cereb Blood Flow Metab 2005;25:763-774.

25 Liu XG, Feng YP: Protective effect of dl-3-n-butylphthalide on ischemic neurological damage and abnormal behavior in rats subjected to focal ischemia (in Chinese). Yao Xue Xue Bao 1995;30:896-903.

-26 Feng YP, Hu D, Zhang LY: Effect of DL-butylphthalide (NBP) on mouse brain energy metabolism in complete brain ischemia induced by decapitation (in Chinese). Yao Xue Xue Bao 1995;30:741-744.

27 Yan CH, Feng YP, Zhang JT: Effects of dl-3-n-butylphthalide on regional cerebral blood flow in right middle cerebral artery occlusion rats. Zhongguo Yao Li Xue Bao 1998;19:117-120.

28 Xiong J, Feng Y: The protective effect of butylphthalide against mitochondrial injury during cerebral ischemia. Yao Xue Xue Bao 2000;35:408-412.

29 Dong GX, Feng YP: Effects of NBP on ATPase and anti-oxidant enzymes activities and lipid peroxidation in transient focal cerebral ischemic rats (in Chinese). Zhongguo Yi Xue Ke Xue Yuan Xue Bao 2002;24:93-97.

-30 Chang Q, Wang XL: Effects of chiral 3-n-butylphthalide on apoptosis induced by transient focal cerebral ischemia in rats. Acta Pharmacol Sin 2003;24:796-804. 